\title{
Assisted crack tip flipping under Mode I thin sheet tearing
}

\author{
Felter, Christian Lotz; Nielsen, Kim Lau
}

Published in:

European Journal of Mechanics A - Solids

Link to article, DOI:

10.1016/j.euromechsol.2017.01.009

Publication date:

2017

Document Version

Peer reviewed version

Link back to DTU Orbit

Citation (APA):

Felter, C. L., \& Nielsen, K. L. (2017). Assisted crack tip flipping under Mode I thin sheet tearing. European Journal of Mechanics A - Solids, 64, 58-68. https://doi.org/10.1016/j.euromechsol.2017.01.009

\section{General rights}

Copyright and moral rights for the publications made accessible in the public portal are retained by the authors and/or other copyright owners and it is a condition of accessing publications that users recognise and abide by the legal requirements associated with these rights.

- Users may download and print one copy of any publication from the public portal for the purpose of private study or research.

- You may not further distribute the material or use it for any profit-making activity or commercial gain

- You may freely distribute the URL identifying the publication in the public portal

If you believe that this document breaches copyright please contact us providing details, and we will remove access to the work immediately and investigate your claim 


\title{
Assisted Crack Tip Flipping under Mode I Thin Sheet Tearing
}

\author{
C.L. Felter, K.L. Nielsen* \\ Department of Mechanical Engineering, Solid Mechanics, Technical University of Denmark, \\ DK-2800 Kgs. Lyngby, Denmark
}

\section{Abstract}

Crack tip flipping, where the fracture surface alternates from side to side in roughly $45^{\circ}$ shear bands, seems to be an overlooked propagation mode in Mode I thin sheet tearing. In fact, observations of crack tip flipping is rarely found in the literature. Unlike the already established modes such as slanting, cup-cone (rooftop), or cup-cup (bathtub) the flipping crack never settles in a steady-state as the near tip stress/strain field continuously change when the flip successively initiates and develops shear-lips. A recent experimental investigation has revealed new insight by exploiting 3D X-ray tomography scanning of a developing crack tip flip. But, it remains to be understood what makes the crack flip systematically, what sets the flipping frequency, and under which material conditions this mode occurs. The present study aims at investigating the idea that a slight out-of-plane action (Mode III type loading) on the tip of a slant Mode I crack can provoke it to flip to the opposite side. Both experiments and micromechanics based modeling support this hypothesis.

Keywords: Thin Sheet, Mode I tearing, Gurson model, Fracture modes, Surface

Morphology

*Tel: +45 4525-4258, Fax: +45 4593-1475

URL: kin@mek.dtu.dk (K.L. Nielsen) 


\section{Introduction}

The fracture surface morphology that develops during Mode I tearing of ductile thin sheet metals depends on both the material microstructure such as size, shape, and distribution of void nucleation sites, as-well as the configuration of existing damage, and the elastic-plastic material properties. Driven by severe plastic deformation, and hydrostatic tension, a continuous process of void nucleation and void growth to coalescence takes place at the leading edge of an advancing crack (Pineau et al., 2016). This process eventually sets the appearance of the crack surface and the crack propagation mode (cup-cup, cup-cone, or slanting). The evolution of a limited number of voids is associated with significant void growth, revealing it-self as large dimples on the fracture surface, and are typically related to severe local thinning of the sheet and crack tip tunneling. In contrast, rapid growth of a large population of micro-voids is commonly seen as smaller dimples, e.g during the slant tearing mode where the crack face has tilted (roughly) $\pm 45^{\circ}$ to the loading direction. In general, low strength metals typically show severe tunneling of the crack tip and favor a cup-cup (bathtub like) tearing mode, whereas crack growth in high strength sheet metals is governed by plastic flow localizing into one of two shear bands traveling with the tip (slanting) (see also Pardoen et al., 2004). The transition from flat to slant has been studied in details by Hickey and Ravi-Chandar (2015). In reality, however, most fracture surfaces show a mixture of propagation modes. For example, the propagation of a slanted crack can be accompanied by large shear-lips near the free sheet surface or complete shear band switches seemingly distributed randomly on the fracture surface. Gruben et al. (2013) observed an occasional shear band switch (or flip) under Mode I tearing of dual-phase 
steel, whereas Rivalin et al. (2001); Simonsen and Törnqvist (2004); Zheng et al. (2009);

El-Naaman and Nielsen (2013) have observed systematic, and successive, flipping of a slant crack in a range of materials. Nielsen and Gundlach (2016) recently exploited X-ray tomography scanning to access the sheet interior and studied the very tip of a slanted crack where a flip is underway. From their study it is clear that the crack tip flipping develops by the growing shear-lips near the outer surface behind the leading slanted tip.

\subsection{Motivation and preliminary experimental findings}

Once the crack slants to a $45^{\circ}$ shear band under a Mode I far-field an out-ofplane action develops due to the loss of symmetry in the system. The hypothesis is that it is this action, in combination with a fortunate set of material properties, which is responsible for the naturally occurring crack tip flip (see also El-Naaman and Nielsen, 2013; Nielsen and Gundlach, 2016). Essentially, the asymmetry in the near tip stress/strain field starts to favor crack propagation in the otherwise inactive shear band as the out-of-plane deflection of the sheet intensifies.

To investigate the effect of a slight out-of-plane action (Mode III type loading) on a propagating Mode I tearing crack, and to gain control of the mixity, a set-up where double edge notched tensile test (DENT) specimens are loaded in combined tension and torsion is considered, see Fig. 1. Samples were cut from $3 \mathrm{~mm}$ rolled 6082-T6 aluminum sheets and heat treated during 16 hours at $180{ }^{\circ} \mathrm{C}$ from a super-saturated solid solution state. Finally, the samples were quenched. With this preparation the samples primarily showed slant fracture over the entire ligament when subject to pure tensile loading (Mode I). Quasi-static conditions were striven for throughout, and in 
all experiments this implied a tensile loading rate of $0.5 \mathrm{~mm} / \mathrm{s}$.

A series of experiments were subsequently carried out under proportional tensile and torsional loading (Mode I/Mode III). This was achieved by prescribing a rotation of the lower gripper on the tensile test machine (Instron). An example of a typical fracture surface morphology is shown in Fig. 2a. Again, a slant fracture surface is obtained, however, this time a rotational symmetry around the axial center axis is observed, because the small imposed torsional loading promotes void growth in one of the two possible shear bands. It is worth to notice that only a very limited rotation (1 $\operatorname{deg} / \mathrm{s})$ was applied.

In a final series of experiments, the direction of the torsional load was inverted halfway through the tests. During the first half the torsional loading rate was $1 \mathrm{deg} / \mathrm{s}$, and during the last half it was $-3 \mathrm{deg} / \mathrm{s}$. The consistent effect was a crack flip as depicted in Fig. 2b. Thus, the experiments show that the out-of-plane action, in fact, can change the active shear band and thereby make a growing slant crack flip. Yet it remains to be clarified what material properties are required for such non-symmetric loading to be able to cause a shear band switch in a crack evolving under far-field Mode I.

In the present work a number of numerical simulations are carried out with the aim of reproducing the qualitative results from the preliminary experimental work, and investigating how the response depends on various model parameters. While direct comparison of e.g. force-displacement curves from the experiments and the simulations could be relevant this would require detailed identification of material parameters, which is a non-trivial and demanding task (see Springmann and Kuna, 2005; Xue 


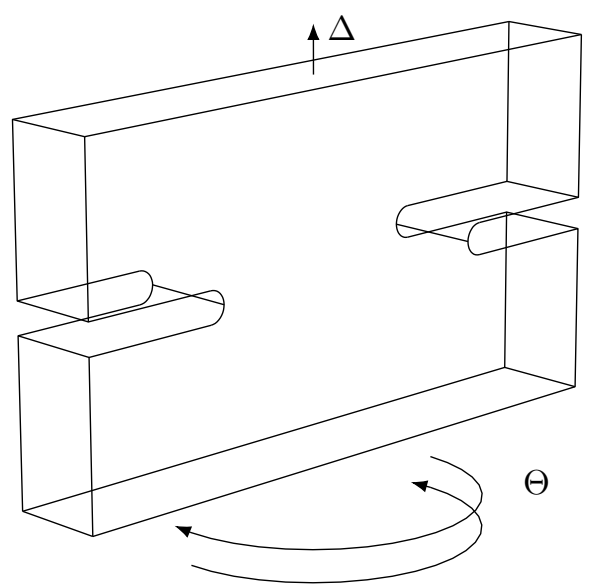

Figure 1: Sketch of experimental setup for DENT specimen subject to displacement $\Delta$ controlled tension and torsion $\Theta$ indicated by arrows. Torsion in the clockwise direction (viewed from above) will be denoted a "positive torsion", while torsion in the opposite direction will be denoted "negative torsion".

et al., 2010) that has not been undertaken. Therefore, in this work, only qualitative comparisons with experiments are possible. Still, within the numerical framework the results are quantified and compared.

\section{Material model}

The presentation is made using general tensor notation, where sub-/super-scripted indices denote co-/contra-variant tensor components, respectively, and ()$_{, i}$ denotes covariant differentiation in the reference frame. The incremental field quantities are denoted by ( ) (Budiansky, 1964; Hutchinson, 1973; Tvergaard, 1990).

The classical micro-mechanics based Gurson model is adopted to represent the void growth to coalescence process that governs the ductile failure of the thin sheets considered. The material yield surface reads

$$
\Phi=\frac{\sigma_{e}^{2}}{\sigma_{M}^{2}}+2 q_{1} f^{*} \cosh \left(\frac{q_{2}}{2} \frac{\sigma_{k}^{k}}{\sigma_{M}}\right)-\left[1+\left(q_{1} f^{*}\right)^{2}\right]=0
$$




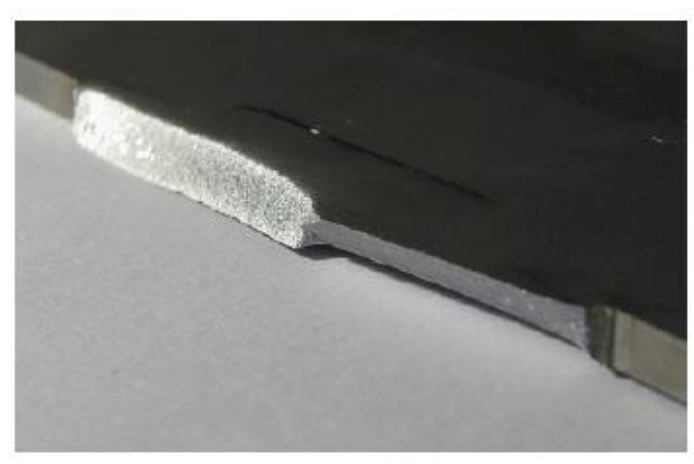

(a)

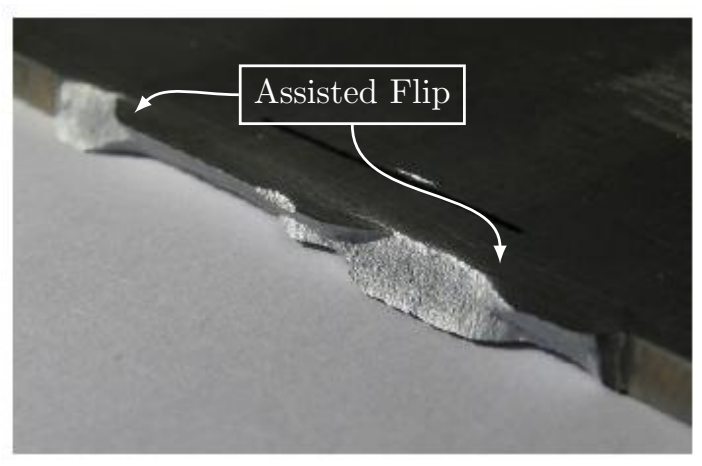

(b)

Figure 2: (a) Fracture surface after combined proportional (tension and torsion type) loading. (b) Fracture surface after steady Mode I loading combined with alternating torsional loading.

with the void coalescence model (Eq. 2) introduced by Tvergaard and Needleman (1984) to accelerate void growth at a critical porosity $f_{c}$. Final fracture takes place at $f^{*}=f_{U}^{*}=1 / q_{1}$

$$
f^{*}(f)= \begin{cases}f & \text { for } f \leq f_{c} \\ f_{c}+\left(f_{U}^{*}-f_{c}\right) \frac{f-f_{c}}{f_{f}-f_{c}} & \text { for } f>f_{c}\end{cases}
$$

The present study assumes a rate-independent material response such that the current material state is characterized by; the reference stress in the matrix material, $\sigma_{M}$, the Cauchy stress that gives the average stress field in the damaged material, $\sigma^{i j}$, the void volume fraction, $f$, which represents the softening effect owing to the evolution of spherical micro-voids. For $f=0$ the material model reduces to that of a conventional J2-flow material. The classical Gurson model is developed for isotropic material behavior such that the effective Mises stress becomes $\sigma_{e}=\sqrt{3 s^{i j} s_{i j} / 2}$, with $s^{i j}=\sigma^{i j}-G^{i j} G_{k l} \sigma^{k l} / 3$ being the Cauchy stress deviator. Here, $G^{i j}$ and $G_{i j}$ are the metric tensors of the convected frame.

Throughout, void nucleation has been omitted to limit the number of model param- 
eters. Thus, the evolution of the void population is solely determined by the growth rate

$$
\dot{f}=(1-f) G^{i j} \dot{\eta}_{i j}^{p}
$$

where $\dot{\eta}_{i j}^{p}$ is the plastic strain increments given by

$$
\dot{\eta}_{i j}^{p}=\frac{1}{H} m_{i j} m_{k l} \stackrel{\nabla}{\sigma} k l
$$

with, $m_{i j}$ being normal to the yield surface, $H$ the hardening modulus, and $\stackrel{\nabla}{\sigma} i j$ the Jaumann stress rate (see Tvergaard, 1990, for details). Plastic yielding requires $\Phi=0$ and $\dot{\Phi}>0$ during elastic deformation, whereas plastic loading continues as long as $\Phi=0$ and $m_{i j} \sigma^{i j} / H \geq 0$. The work on the microscopic and macroscopic levels are equated such that $(1-f) \sigma_{M} \dot{\varepsilon}_{M}^{p}=\sigma^{i j} \dot{\eta}_{i j}^{p}$, which together with $\dot{\varepsilon}_{M}^{p}=\left(\frac{1}{E_{t}}-\frac{1}{E}\right) \dot{\sigma}_{M}$ yields the microscopic reference stress rate

$$
\dot{\sigma}_{M}=\frac{E E_{t}}{E-E_{t}} \frac{\sigma^{i j} \dot{\eta}_{i j}^{p}}{(1-f) \sigma_{M}} .
$$

Here, $E$ is the Young's Modulus and $E_{t}$ is the tangent modulus. The material hardening curve (in terms of true stress and logarithmic strain) under uni-axial tension of the matrix material is approximated by a power-law hardening material such that

$$
\varepsilon=\left\{\begin{array}{lll}
\frac{\sigma_{M}}{E} & \text { for } & \sigma_{M}<\sigma_{\mathrm{y}} \\
\frac{\sigma_{\mathrm{y}}}{E}\left(\frac{\sigma_{M}}{\sigma_{\mathrm{y}}}\right)^{1 / N} & \text { for } & \sigma_{M} \geq \sigma_{\mathrm{y}}
\end{array}\right.
$$

Here, $N$ is the strain hardening exponent and $\sigma_{\mathrm{y}}$ is the initial yield stress. The power hardening law is chosen for simplicity and to limit the number of model parameters.

\section{Numerical model}

A 3D finite element code that accounts for finite strains and finite deformations using a total Lagrangian formulation is developed from the dynamic form of the principle of 
virtual work

$$
\int_{V} \tau^{i j} \delta \eta_{i j} \mathrm{~d} V=\int_{S} T^{i} \delta u_{i} \mathrm{~d} S-\int_{V} \rho \frac{\partial^{2} u^{i}}{\partial t^{2}} \delta u_{i} \mathrm{~d} V
$$

with

$$
\eta_{i j}=\frac{1}{2}\left(u_{i, j}+u_{j, i}+u_{i}^{k} u_{k, j}\right)
$$

Here, $\tau^{i j}$ are the contravariant components of the Kirchhoff stress, $\eta_{i j}$ are the covariant components of the Lagrangian strain, $\rho$ is the material mass density, and $V$ and $S$ are the volume and surface of the domain in the reference configuration.

An in-house FE-code is written in Fortran 2008 with OpenMPI (Gabriel et al., 2004) for parallel execution on an arbitrary number of CPUs. The partitioning of the mesh is carried out using the software package METIS (Karypis and Kumar, 1999). Note that the overall scheme is fully explicit and consequently there is no global system equation. The stable time step is proportional to the Courant-Friedrichs-Lewi-number and no artificial scaling of mass is used in the simulations.

The analysis uses 20-noded isoparametric solid elements with reduced $(2 \times 2 \times 2)$ Gauss point quadrature for all integrals, except for the mass matrix which requires full $(3 \times 3 \times 3)$ Gauss integration. The equations of motion, i.e. the momentum equations, are time-integrated using an explicit Newmark $\beta$-method.

The Gurson yield surface shrinks in stress space as damage (the void volume fraction f) evolves in the material, and thus the material looses stress carrying capacity. The damage evolution in a Gauss point that reaches $f=0.99 f_{f}$ is turned off in order to maintain numerical stability. When two Gauss points in an element satisfies this criteria, the element is deleted and any residual forces on neighboring elements are ramped down over a short period of time (see also Tvergaard, 1982). 


\section{Simulations}

A geometry and loading equivalent to the one in Jensen (2015) is applied for the simulations, i.e. the regions inside the grippers (of the tensile test apparatus) are considered rigidly clamped and are therefore not modeled, and by splitting the prescribed displacements into contributions on the upper and lower part a high degree of symmetry is achieved (see Fig. 3a). These, so-called rotational symmetries, are applicable when the solution fields (e.g. displacements, nodal forces, etc.) are invariant with respect to a $180^{\circ}$ rotation about say the $x^{3}$-axis. With Fig. 4 in mind, a simple relation develops such that the forces on a node "A" on the boundary of the computational domain can be determined by

$$
F_{i}^{\mathrm{A}}=f_{i}^{\mathrm{a}, 2}+f_{i}^{\mathrm{b}, 1}+\Psi_{i}\left(f_{i}^{\mathrm{c}, 1}+f_{i}^{\mathrm{b}, 2}\right)
$$

where $\boldsymbol{\Psi}=[-1,-1,1]$ and subscript $i=1,2,3$ indicates Cartesian components (see also Nielsen, 2008, 2010). Similarly, the total (lumped) mass at node "A" becomes; $M^{\mathrm{A}}=m^{\mathrm{a}, 2}+m^{\mathrm{b}, 1}+m^{\mathrm{c}, 1}+m^{\mathrm{b}, 2}$. In all simulations two such anti-symmetric boundary conditions are introduced on the $\left(x^{1}, x^{2}\right)$-plane and the $\left(x^{2}, x^{3}\right)$-plane, respectively, (see Fig. 3b) in order to reduce the computational cost by a factor of roughly 4 . The combined tension and torsional loading is imposed by prescribing a rigid body motion of the nodes on the plane through $(0,0,-H / 2)$ parallel to the $\left(x^{1}, x^{2}\right)$-plane. All accelerations are introduced gradually using a smooth step function for the displacement rates.

Referring to the sketch in Fig. 3a and parameters marked by $\left(^{\dagger}\right)$ in Table 1 a dimensional analysis yields the following dimensionless groups

$$
\Phi=F\left(\frac{H}{b}, \frac{B}{b}, \frac{T}{b}, \frac{r}{b}, \frac{\dot{\Theta}}{\dot{\Delta} / b}, \frac{\dot{\Delta}}{\sqrt{E / \rho}}, \nu, f_{0}, N, \frac{\sigma_{\mathrm{y}}}{E}\right)
$$




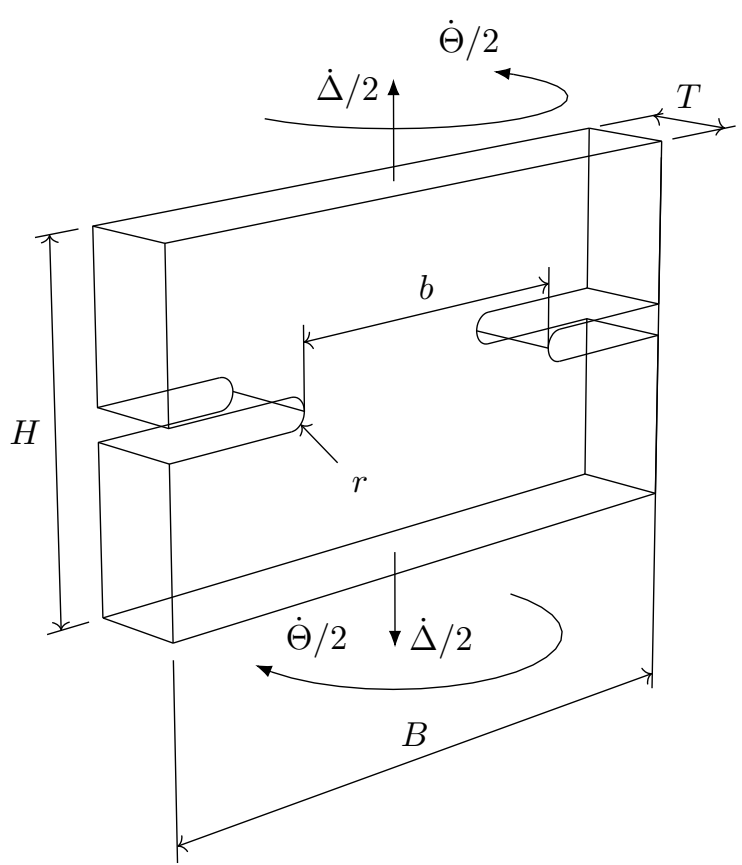

(a)

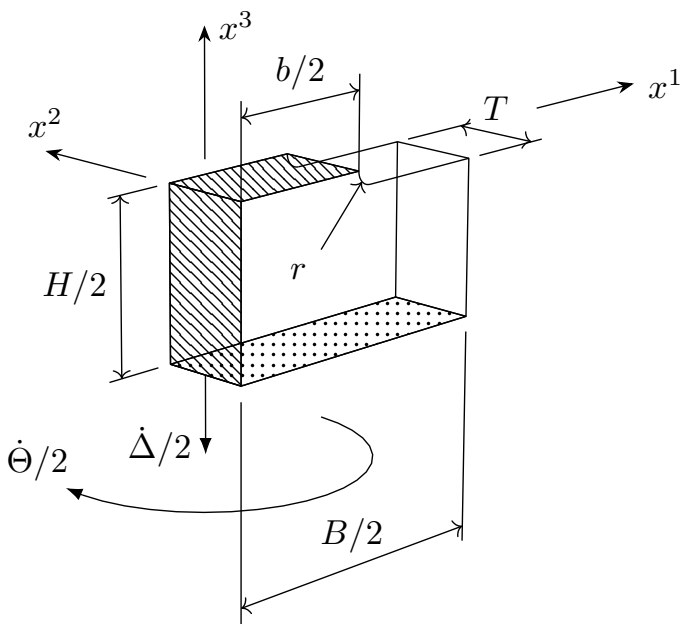

(b)

Figure 3: (a) Truncated geometry and modified loading (torsion add translation applied on both upper and lower part) for modeling. (b) Simulation domain taking advantage of symmetries in order to reduce model size. Hatched: Anti-symmetric boundary conditions applied. Dotted: Controlled rigid body motion.

The first four groups are tied to the specimen dimensions which will be kept constant for all simulations. The fifth group describes the ratio between Mode III and Mode I, which will also be treated the same in all simulations (close to that of the preliminary experimental study in Section 1.1). Finally, the Poisson's ratio $\nu$ is fixed at 0.3 which is an appropriate value for most metals. This leaves four dimensionless groups to be examined: $\dot{\Delta} / \sqrt{E / \rho}$ is the ratio between loading speed and speed of sound in the material, $f_{0}$ is the initial void volume ratio, $N$ is the strain hardening exponent, and $\sigma_{\mathrm{y}} / E$ is the initial yield stress.

The Gurson model is inherently mesh dependent, and requires an element size 


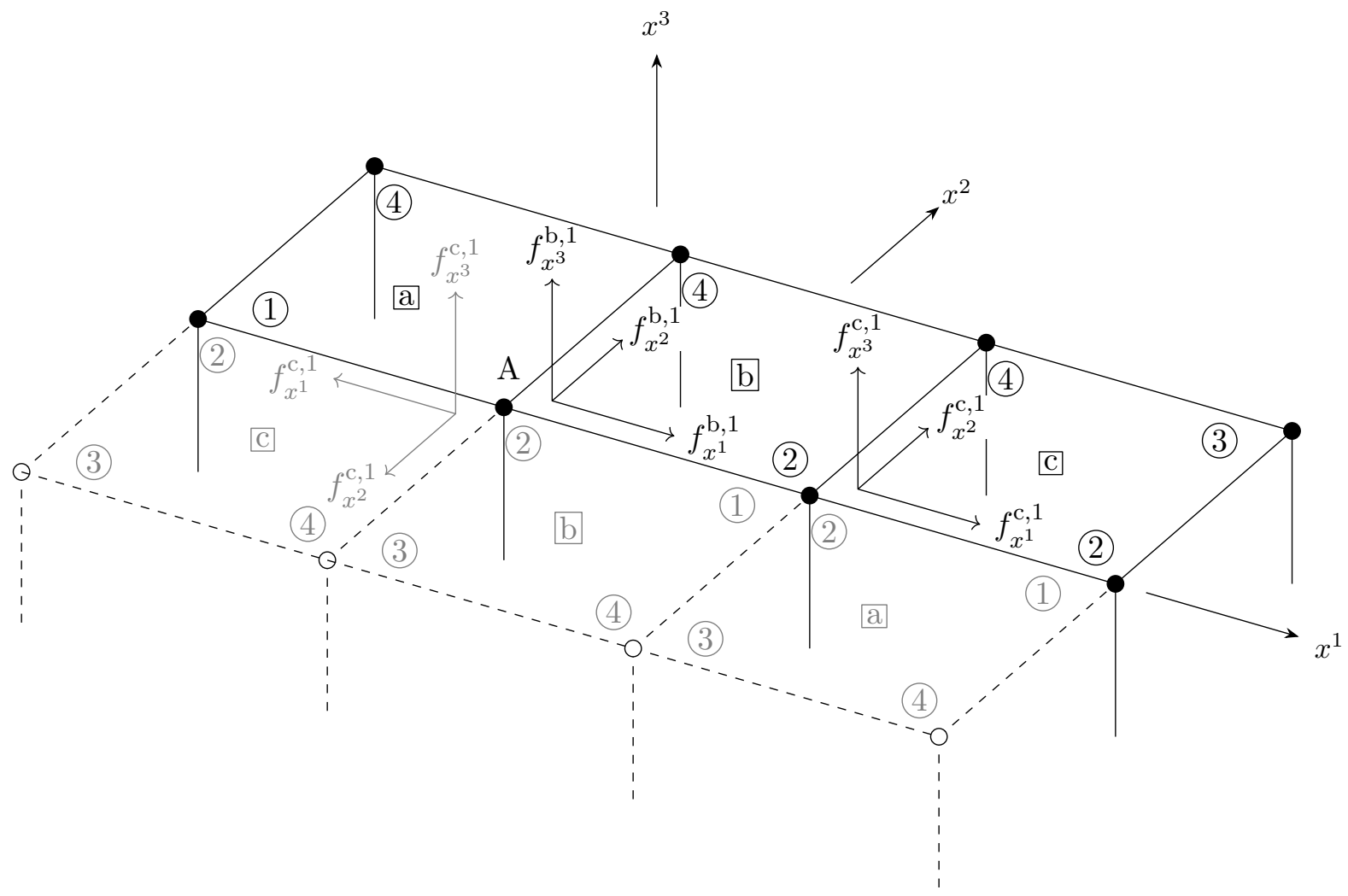

Figure 4: Illustration of the anti-symmetric boundary condition. Full lines: Original mesh, Dashed lines: Mesh after a $180^{\circ}$ rotation around the $x^{3}$-axis, Letters in boxes: Element label, Numbers in circles: Node number (local numbering). Note that some items (forces components, node numbers, etc) are left out for the sake of a clear representation. 
comparable to the dominant void spacing in the material $(\sim 100 \mu \mathrm{m})$ to ensure an accurate representation of ductile failure. Thus, a plate of a few millimeters thickness demands a significant amount of elements - in particular when aiming for propagating the crack several plate thicknesses. In all simulations 32 elements through the plate thickness are used. The total number of elements is 70,376 which in itself puts a constraint on the parametric study, and the number of time steps is on the order of 3,000,000. One simulation takes roughly 15 hours on a computer cluster with 200 CPUs (10 nodes of dual Intel Xeon E5-2680v2 with 10 cores).

\section{Results}

First and foremost, the simulations include a reference case as specified in Table 1, which serves as basis for the parametric investigation. Properties marked with $\left(^{*}\right)$ in Table 1 are modified systematically in the following sections, while all other properties remain unchanged, and the Mode I loading rate is set such that the final displacement of the boundaries always becomes $\Delta / H=0.056$. The prescribed torsional load (see Fig. 1) is composed of two parts; first the torsion is in the positive direction, followed by a twist in the negative direction (at double rate). The event when the torsional loading changes sign is denoted "Changeover" in Table 1. This loading scheme is similar to that used in the experimental work by Jensen (2015).

\subsection{Influence of deformation rate $\dot{\Delta} / \sqrt{E / \rho}$}

A single experiment takes a couple of minutes in the lab, however, the deformation is simulated to take place over a few milli-seconds to save computational time. This is achieved by scaling the loading rates by a sufficiently large factor $\alpha$, such that 


\begin{tabular}{|c|c|c|}
\hline Material parameter & Notation & Value \\
\hline Young's modulus ${ }^{\dagger}$ & $E$ & $70 \mathrm{GPa}$ \\
\hline Yield stress ${ }^{\dagger *}$ & $\sigma_{\mathrm{y}}$ & $210 \mathrm{MPa}$ \\
\hline Poisson's ratio ${ }^{\dagger}$ & $\nu$ & 0.3 \\
\hline Strain Hardening exponent ${ }^{\dagger *}$ & $N$ & 0.05 \\
\hline Initial void volume fraction $^{\dagger *}$ & $f_{0}$ & 0.01 \\
\hline Critical void volume fraction & $f_{\mathrm{c}}$ & 0.2 \\
\hline Final void volume fraction & $f_{\mathrm{f}}$ & 0.4 \\
\hline Mass density $^{\dagger}$ & $\rho$ & $2700 \mathrm{~kg} / \mathrm{m}^{3}$ \\
\hline Tvergaard parameter 1 & $q_{1}$ & 1.5 \\
\hline Tvergaard parameter 2 & $q_{2}$ & 1.0 \\
\hline Geometry & Notation & Value \\
\hline Plate height ${ }^{\dagger}$ & $\mathrm{H}$ & $45 \mathrm{~mm}$ \\
\hline Plate width ${ }^{\dagger}$ & B & $80 \mathrm{~mm}$ \\
\hline Plate thickness ${ }^{\dagger}$ & $\mathrm{T}$ & $3 \mathrm{~mm}$ \\
\hline Ligament width ${ }^{\dagger}$ & $\mathrm{b}$ & $40 \mathrm{~mm}$ \\
\hline Notch radius ${ }^{\dagger}$ & $\mathrm{r}$ & $0.5 \mathrm{~mm}$ \\
\hline Prescribed deformation & Notation & Value \\
\hline Max tensile displacement & $\Delta_{\max }$ & $2.5 \mathrm{~mm}$ \\
\hline Tensile displacement rate ${ }^{\dagger}$ & $\dot{\Delta}$ & (dependent quantity) \\
\hline Torsional displacement rate ${ }^{\dagger}$ & $\dot{\Theta}$ & (dependent quantity) \\
\hline Proportional loading 1 & $\dot{\Delta} / \dot{\Theta}$ & $1.0 \mathrm{~mm} / \mathrm{deg}$ \\
\hline Proportional loading 2 & $\dot{\Delta} / \dot{\Theta}$ & $-0.5 \mathrm{~mm} / \mathrm{deg}$ \\
\hline Changeover $1 \rightarrow 2$ & $\Delta_{\text {inv }} / \Delta_{\max }$ & 0.36 \\
\hline Displacement rate scaling* & $\alpha=t_{\text {end }}^{\text {experiment }} / t_{\text {end }}^{\text {simulation }}$ & $1 / 3 \cdot 10^{5}$ \\
\hline
\end{tabular}

Table 1: Parameters for the reference case. ${ }^{\dagger}$ Material parameters included in dimensional analysis.

* Parameters that are changed in parametric study. 
$\dot{\Delta}=\alpha \dot{\Delta}^{\text {experiment }}$ and $\dot{\Theta}=\alpha \dot{\Theta}^{\text {experiment }}$. Obviously, this increase in loading rate comes with the price of dynamic effects and increased kinetic energy. However, it is not the intention to enter the regime where dynamics plays a significant role, but rather is it the intention to reproduce the real quasi-static experiments by simulation. Thus, the first parameter to be investigated is the deformation rate and Table 2 displays the different load rate scaling factors considered together with an indication of the type of fracture surface morphology observed. Indeed, by increasing $\alpha$ to $2 \cdot 10^{5}$ the fracture surface morphology changes from a clear crack tip flip to a pure slant fracture. In Figs. 5-6 the corresponding tensile force and the torque is shown $\left(A_{0}\right.$ is the initial ligament cross-section area $b \times T)$. While the tensile force is little affected by the loading speeds considered, large oscillations are observed in the torque, in particular for $\alpha \geq 1 \cdot 10^{5}$. For $\alpha=1 / 2 \cdot 10^{5}$ and $\alpha=1 / 3 \cdot 10^{5}$, the torque curves are nearly identical with the latter value of $\alpha$ producing a rather smooth response. The kinetic energy is in this case less than $2 \%$ of the total energy in the system, throughout the simulation, and it is therefore reasonable to use $\alpha=1 / 3 \cdot 10^{5}$ in the remaining simulations to mimic quasi-static conditions.

It is worth noticing that the predicted crack tip flipping, though assisted by torsional loading, is the first ever reproduced in a numerical model (to the best of the authors knowledge). In Fig. 7, the evolution of the crack is investigated by displaying snapshots of the DENT specimen and contours the void volume fraction $f$ side-by-side. The contours are drawn for $f=0.026$, thereby tracking the front of the damaged zone near the crack. At displacement $\Delta / H=0.0102$ the damage level for $f=0.026$ is just becoming visible at the root of the notches. The next two frames at $\Delta / H=0.0157$ 
and $\Delta / H=0.0213$ show how the initially diffuse damage has become non-symmetric due to the imposed torsional loading. In fact, localization of damage in a single shear band is clearly visible. The changeover for the torsional load happens at a tensile displacement of $\Delta / H=0.02$, and is marked as a dotted line in Figs. 5-6. The effect is visible in the frame belonging to $\Delta / H=0.0269$, where the previously inactive shear band now becomes the dominant one. The end result is a crack tip flip as seen on the last frame at $\Delta / H=0.0435$.

\begin{tabular}{c|c|c|c|c} 
Displacement rate scaling $\alpha$ & $1 / 3 \cdot 10^{5}$ & $1 / 2 \cdot 10^{5}$ & $1 \cdot 10^{5}$ & $2 \cdot 10^{5}$ \\
\hline Crack Propagation Mode & Flip & Flip & Flip & Slant
\end{tabular}

Table 2: Load cases for demonstrating the influence of deformation rate. Other model parameters are as given in Table 1.

\subsection{Influence of initial void volume fraction $f_{0}$}

In this section the initial void volume fraction $f_{0}$ of the material is varied systematically taking the values in Table 3, while all other parameters remain fixed at the values of the reference case (Table 1). By doing so, three different crack surface morphologies are predicted.

When $f_{0}$ belongs to a small interval around $0.01-0.012$, the crack tip flip is observed (see Fig. 8c). The imposed positive torsional load causes a $+45^{\circ}$ slant crack to develop, which is following by a $-45^{\circ}$ slant crack due to reversal of the "twisting" motion. However, the exact location of the crack tip flip is influenced by the initial void volume fraction, and increasing $f_{0}$ is observed to delay the crack tip flip (see Fig. 8d). In these cases, the crack advance, and preceding damage evolution, takes place at a speed which 


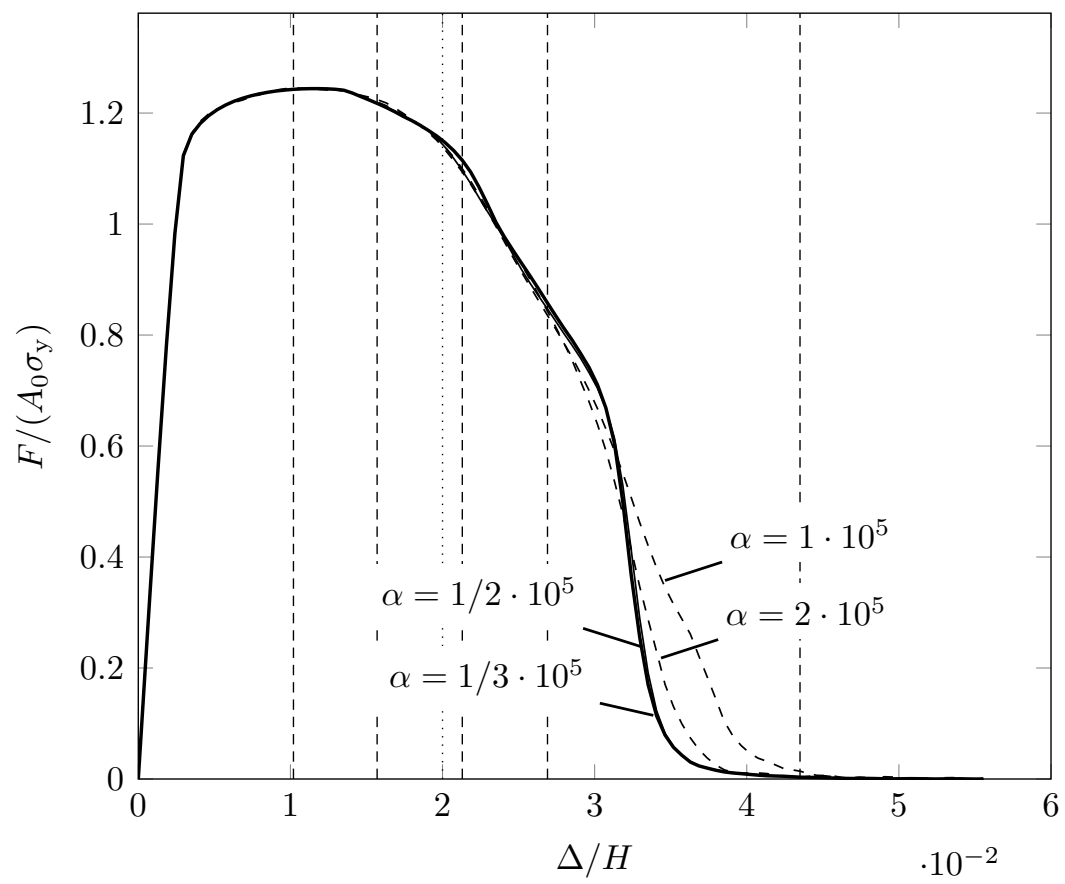

Figure 5: Normalized tensile force for varying loading rates $\alpha$, keeping all other model parameters constant with values given by Table 1 . Dashed vertical lines correspond (from left to right) the displacements indicated in Fig. 7 (top to bottom row). The dotted vertical line indicates the event where the imposed torsional loading changes sign. 


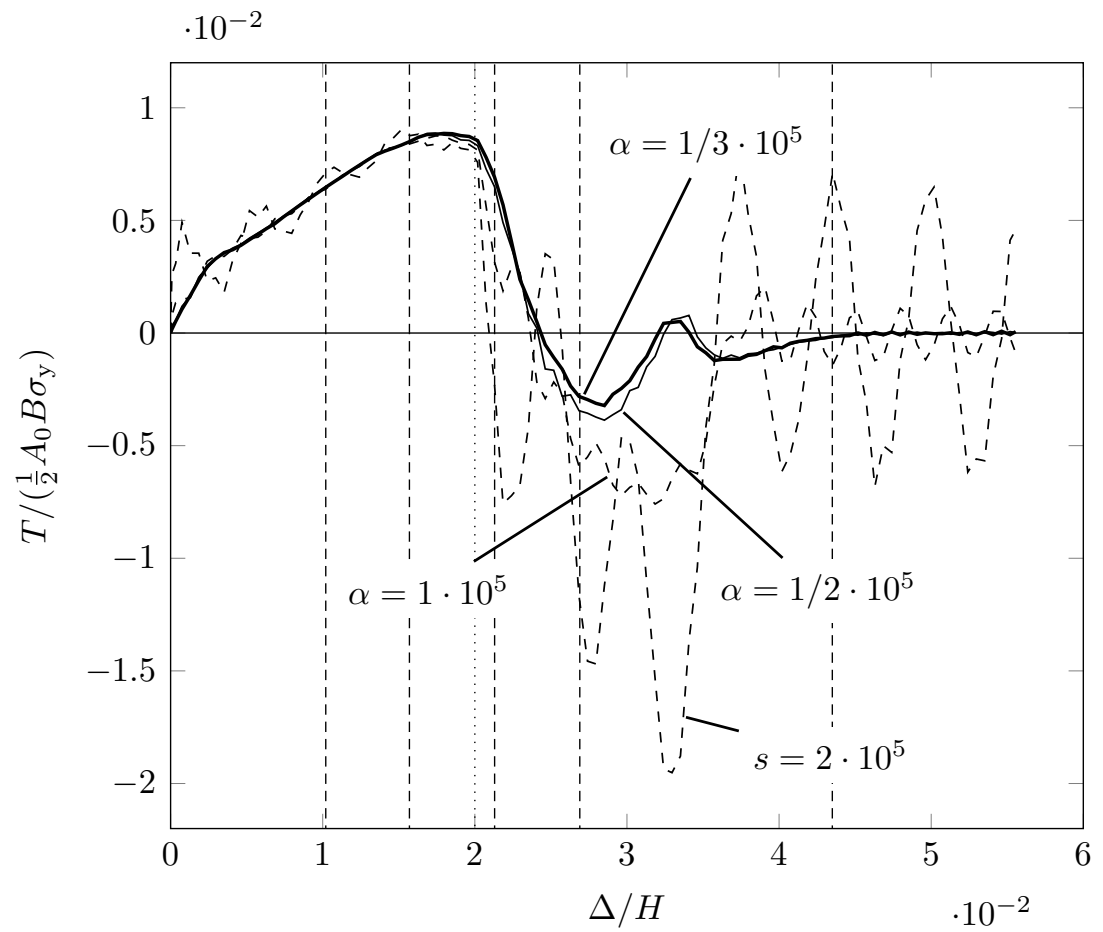

Figure 6: Normalized torque for varying loading rates $\alpha$, keeping all other model parameters constant with values given by Table 1. Dashed vertical lines correspond (from left to right) the displacements indicated in Fig. 7 (top to bottom row). The dotted vertical line indicates the event where the imposed torsional loading changes sign. 


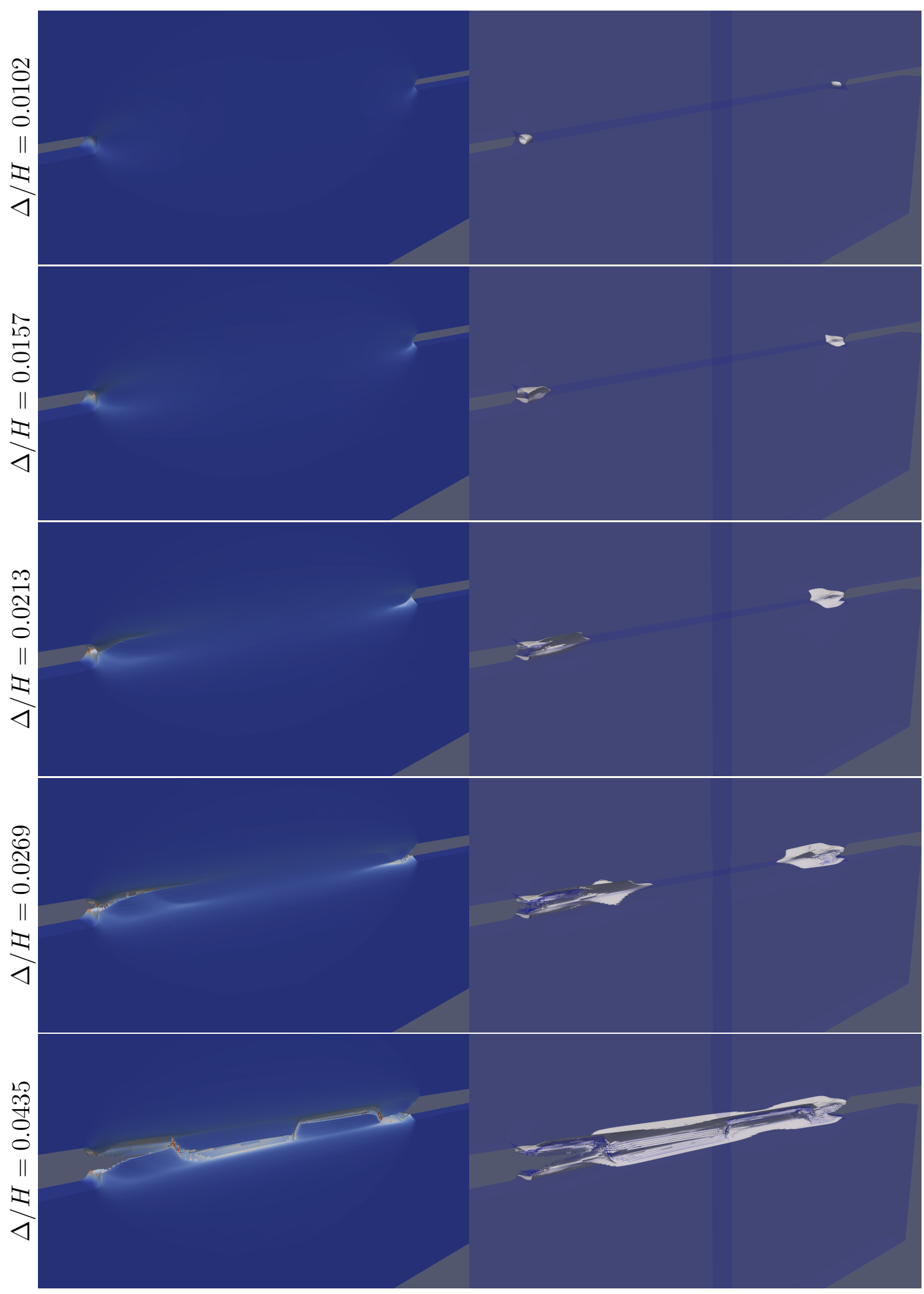

Figure 7: Left: The DENT specimen at increasing tensile (and torsional) displacement colored by effective plastic strain. Right: Contours for void volume fraction $f=0.026$ with transparent geometry. The planes with stronger shading are due to "copy + rotate + paste" of the computational domain, in order to show not only the computational domain, which is merely one-quarter of the real domain. 
enables the crack to react on the out-of-plane action much as it is expected to happen for a naturally occurring crack tip flip.

However, as $f_{0}$ becomes larger more rapid damage evolution takes place and the first positive twist induces a $+45^{\circ}$ slant crack that propagates across the entire ligament. At the time the second negative torsion is imposed damage is already severe (see Fig. 8e), and the negative torsion is therefore not sufficient to flip the crack tip. On the other hand, if $f_{0}$ becomes too small (i.e. below 0.01 ), the fracture surface is composed of two parts. The first part is a cup-cup fracture, whereas the second part displays a $-45^{\circ}$ slant crack due to the negative torsion (see Fig. $8 \mathrm{a}-8 \mathrm{~b}$ ). This is owed to damage evolution being too slow, and the first positive torsion is therefore not sufficient to induce any preference for one shear band over the other.

The tensile force and torque for various values of the initial void volume fraction are shown in Figs. 9-10. As expected, by lowering the initial void volume fraction the tensile force curve shows a higher load carrying capacity of the material and lower initial void growth rate. Both the peak load and the ductility of the specimen are affected. Similarly, the torque displays an increased maximum level when lowering the void volume fraction.

For $f_{0}=0.02$ it is worth noticing that the torque curve drops before the torsional loading is reversed (at $\Delta / H=0.02$ ). This means that the ligament is severely damaged and cannot sustain the imposed torque. Moreover the biggest negative torque, out of all cases investigated, is also found for $f_{0}=0.02$ (see Fig. 10). This reflects much underdeveloped damage in the second shear band as the specimen holds its initially +45 slant crack throughout the fracture surface. 

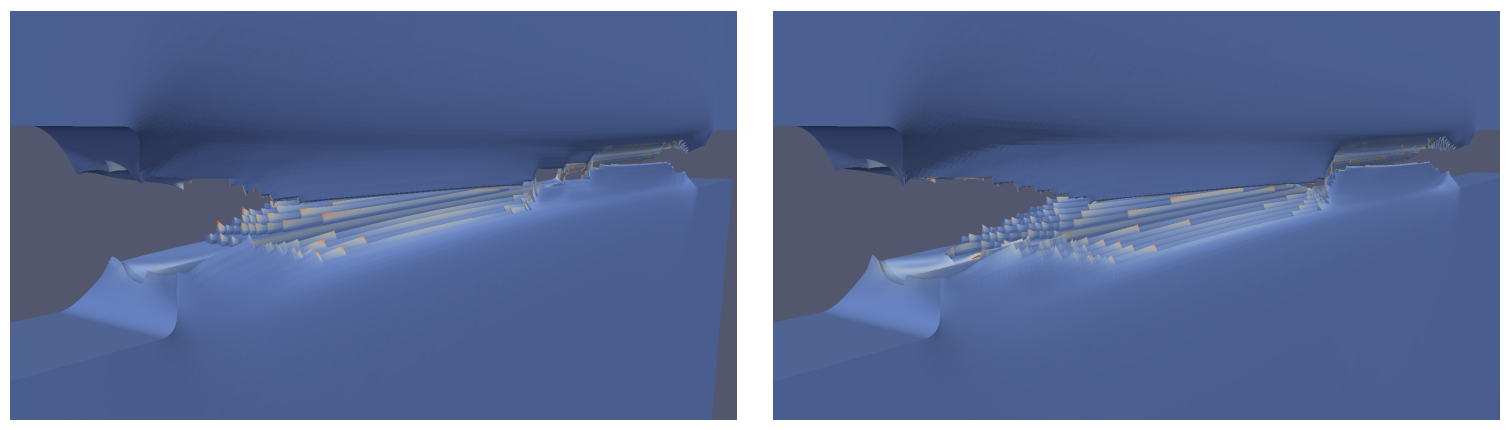

(a) $f_{0}=0.004$

(b) $f_{0}=0.008$
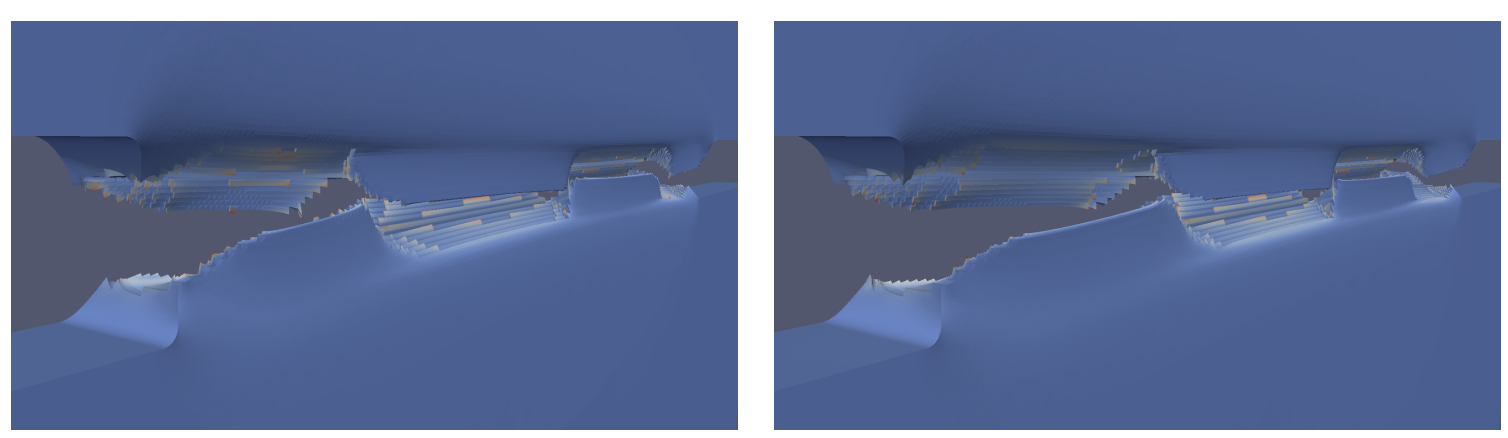

(c) $f_{0}=0.01$ (Reference Case)

(d) $f_{0}=0.012$

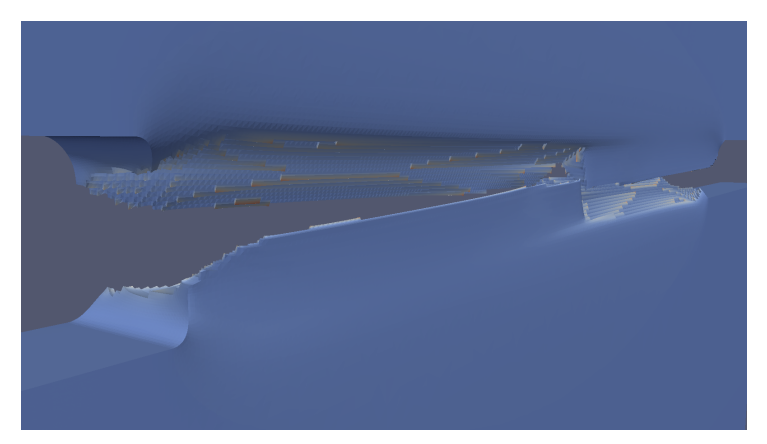

(e) $f_{0}=0.02$

Figure 8: Crack surfaces at end of simulation $(\Delta=0.025)$ for different initial void volume fractions $f_{0}$. Other model parameters are as given in Table 1. 


\begin{tabular}{l|l|c}
$f_{0}$ & Crack Propagation Mode & Fig. \\
\hline 0.004 & Cup-Cup followed by Slant $-45^{\circ}$ & $8 \mathrm{a}$ \\
0.008 & Cup-Cup followed by Slant $-45^{\circ}$ & $8 \mathrm{~b}$ \\
0.01 & Flip (centered, Reference Case) & $8 \mathrm{c}$ \\
0.012 & Flip (late) & $8 \mathrm{~d}$ \\
0.02 & Slant $+45^{\circ}$ across ligament & $8 \mathrm{e}$
\end{tabular}

Table 3: Load cases for the influence initial void fraction $f_{0}$. Other model parameters are as given in Table 1.

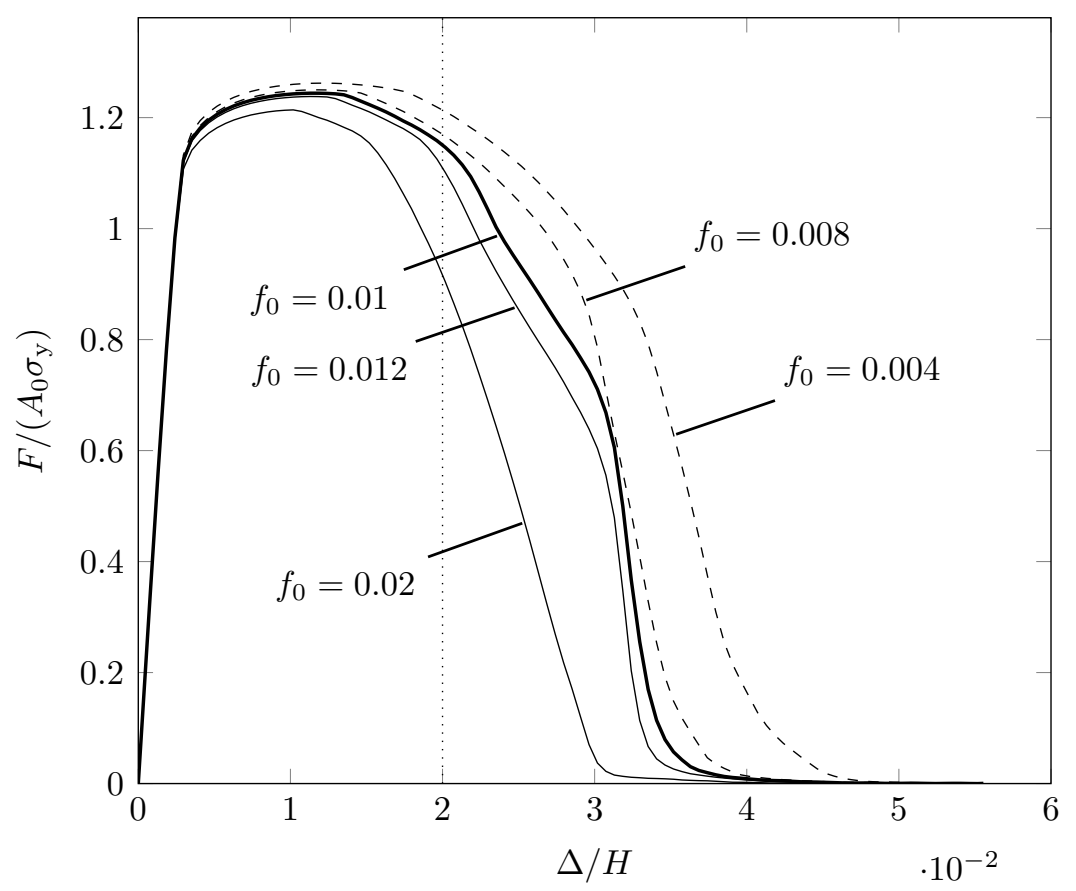

Figure 9: Normalized tensile force for different initial void volume fractions $f_{0}$. All other parameters are as given in Table 1. The vertical dotted line indicates the event where the imposed torsional loading changes sign. 


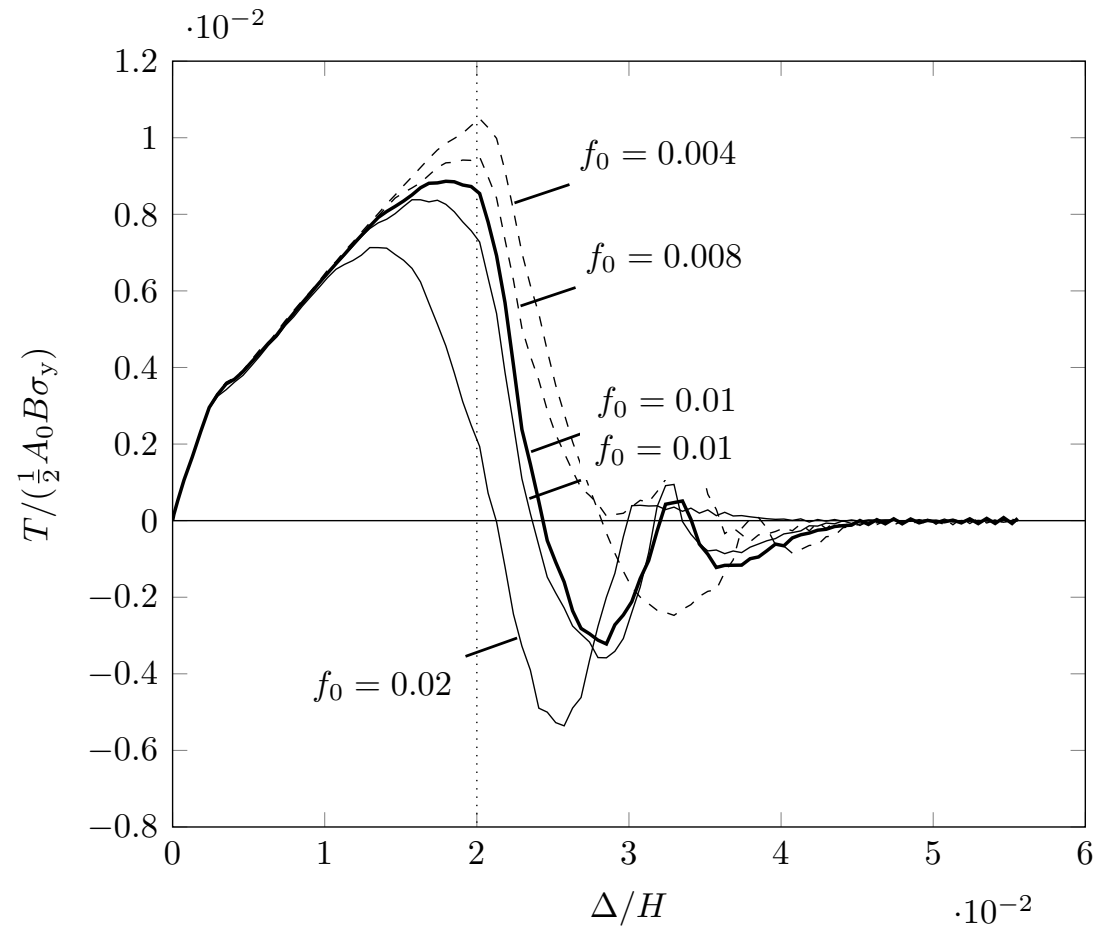

Figure 10: Normalized torque for different initial void volume fractions $f_{0}$. All other parameters are as given in Table 1. The vertical dotted line indicates the event where the imposed torsional loading changes sign. 


\subsection{Influence of the initial yield strain $\sigma_{\mathrm{y}} / E$}

The plate's bending stiffness works against the out-of-plane deflection, while the initial plastic deformation is largely governed by the initial yielding stress. Thus, one dimensionless group of key interest is the initial yield strain $\sigma_{\mathrm{y}} / E$.

In the following set of simulations, the initial yield strain is varied using the values in Table 4. In all simulations Young's Modulus $E$ is kept constant to leave the dynamics of the problem unchanged (the density $\rho$ is also kept constant). Thus, the dimensionless group is varied by changing the initial yield stress $\sigma_{y}{ }^{1}$

The crack surface morphology is shown in Fig. 11 for the cases listed in Table 4 except for the reference case, which is shown in Fig. 8c. It is seen that by lowering the initial yield strain, and thereby increasing the relative stiffness of the plate, the crack tip flipping mechanism engages at an earlier state, and vice verse. In fact, by imposing a sufficiently high value of initial yield strain no flipping is found on the entire ligament. Instead, the crack remains slanted in the +45 degree shear band. This result aligns with the experimental study conducted by Simonsen and Törnqvist (2004) and El-Naaman and Nielsen (2013), where a higher flipping frequency is seen for stiffer systems.

Figures 12 and 13 show the tensile force and torque curves, respectively. Note, that the force and torque are normalized against the current value of initial yield stress $\sigma_{\mathrm{y}}$, whereas the deformation $\Delta / H$ is maintained for better comparison of the curves. Both families of curves show similar features and are largely comparable despite the large

\footnotetext{
${ }^{1}$ To check the dimensional analysis additional simulations (not included) with constant yield strain, $\sigma_{\mathrm{y}} / E$, for different $\sigma_{\mathrm{y}}$ and $E$ have been conducted, indeed showing no change in the model response.
} 


\begin{tabular}{c|l|c}
$\sigma_{\mathrm{y}} / E$ & Crack Propagation Mode & Fig. \\
\hline 0.001 & Flip (early) & $11 \mathrm{a}$ \\
0.002 & Flip & $11 \mathrm{~b}$ \\
0.003 & Flip (centered, Reference Case) & $8 \mathrm{c}$ \\
0.004 & Flip (late) & $11 \mathrm{c}$ \\
0.005 & Slant $+45^{\circ}$ across ligament & $11 \mathrm{~d}$
\end{tabular}

Table 4: Load cases for the influence of initial yield stress $\sigma_{\mathrm{y}} / E$. Other model parameters are as given in Table 1.

interval covered by $\sigma_{\mathrm{y}}$.

\subsection{Influence of the strain hardening exponent $N$}

The thinning of the plate in front of the advancing crack tip is largely controlled by the material strain hardening and, moreover, the strain hardening must be expected to affect the out-of-plane deflection. Thus, the material strain hardening $N$ is another dimensional group of key interest in understanding the crack tip flipping mechanism.

Figures 14a-14d show the result of applying the hardening exponents given in Table 5. It is remarkable how these results look similar to the ones for varying initial void volume fraction, expect that small (high) $N$ corresponds to high (small) $f_{0}$. Also, the force and torque curves in Figs. 15-16 share strong similarities with the corresponding curves for varying $f_{0}$. The simulations suggest that the strain hardening exponent is a very important parameter for the crack tip flipping mechanism, since changes of only $\pm 10 \%$ have a dramatic effect on the fracture surface. 


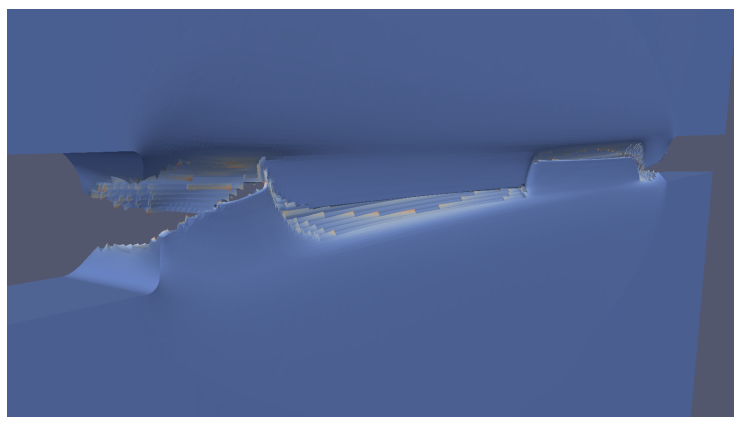

(a) $\sigma_{\mathrm{y}} / E=0.001$

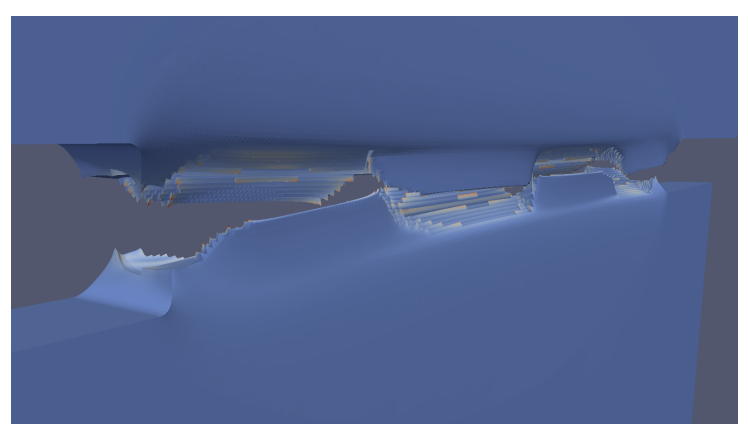

(c) $\sigma_{\mathrm{y}} / E=0.004$

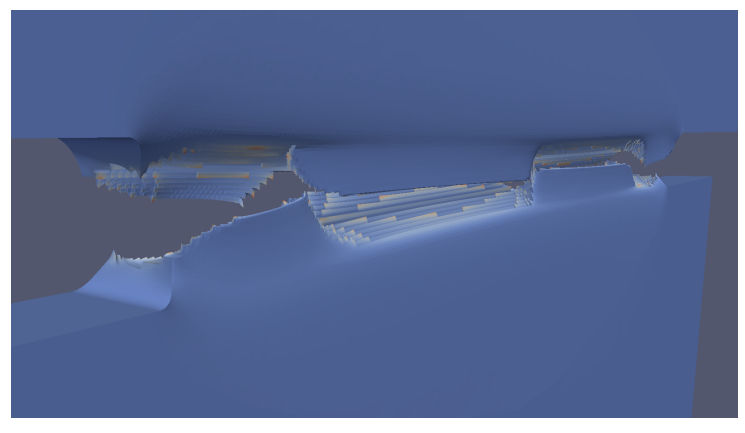

(b) $\sigma_{\mathrm{y}} / E=0.002$

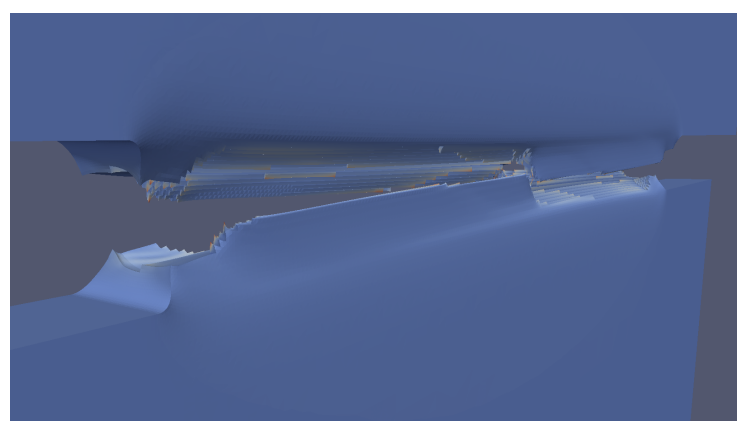

(d) $\sigma_{\mathrm{y}} / E=0.005$

Figure 11: Crack surface morphology for different initial yield strains $\sigma_{\mathrm{y}} / E$. Other model parameters are as given in Table 1.

\begin{tabular}{l|l|c}
$N$ & Crack Propagation Mode & Fig. \\
\hline 0.04 & Slant $+45^{\circ}$ & $14 \mathrm{a}$ \\
0.045 & Flip (late) & $14 \mathrm{~b}$ \\
0.05 & Flip (centered, Reference Case) & $8 \mathrm{c}$ \\
0.055 & Cup-Cup followed by Flip (early) & $14 \mathrm{c}$ \\
0.06 & Cup-Cup followed by Slant $-45^{\circ}$ & $14 \mathrm{~d}$
\end{tabular}

Table 5: Load cases for the influence of strain hardening exponent $N$. Other model parameters are as given in Table 1. 


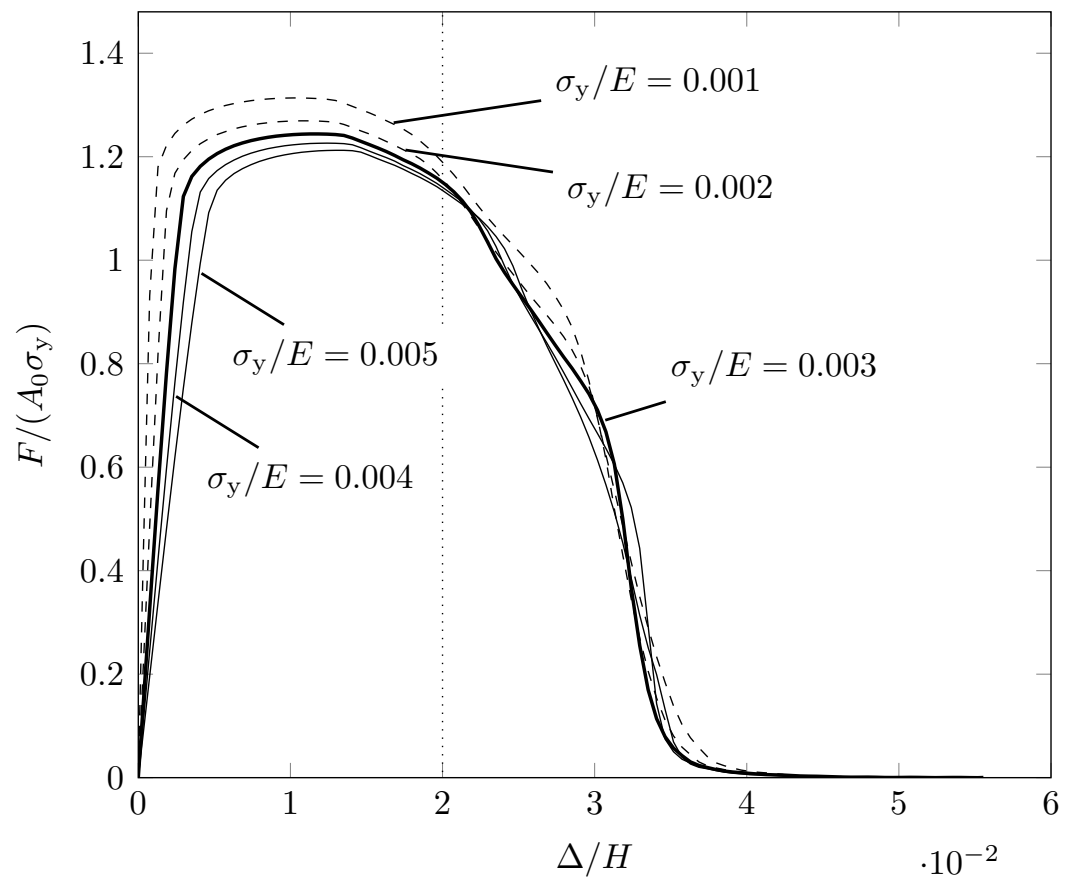

Figure 12: Normalized tensile force for different initial yield strains $\sigma_{\mathrm{y}} / E$. All other parameters are as given in Table 1. The vertical dotted line indicates the event where the imposed torsional loading changes sign.

\section{Concluding remarks}

A number of full 3D numerical simulations have been carried out by the use of Gurson's material model with the aim of (1) Qualitatively replicate experimental results for assisted crack tip flipping in a DENT type specimen, (2) Determine the influence of material parameters on the crack tip susceptibility.

The parametric study shows that crack tip flipping is likely to occur only for certain materials as the parameter span that shows flipping is fairly narrow. This is, however, not surprising as systematic and repeated flipping is rarely encountered in experiments. From the simulation results it is found that the initial void volume fraction and the strain hardening both are parameters that strongly affect the crack tip flip, but they have opposite effects: Increasing $f_{0}$ will delay the crack tip flip, while increasing $N$ 


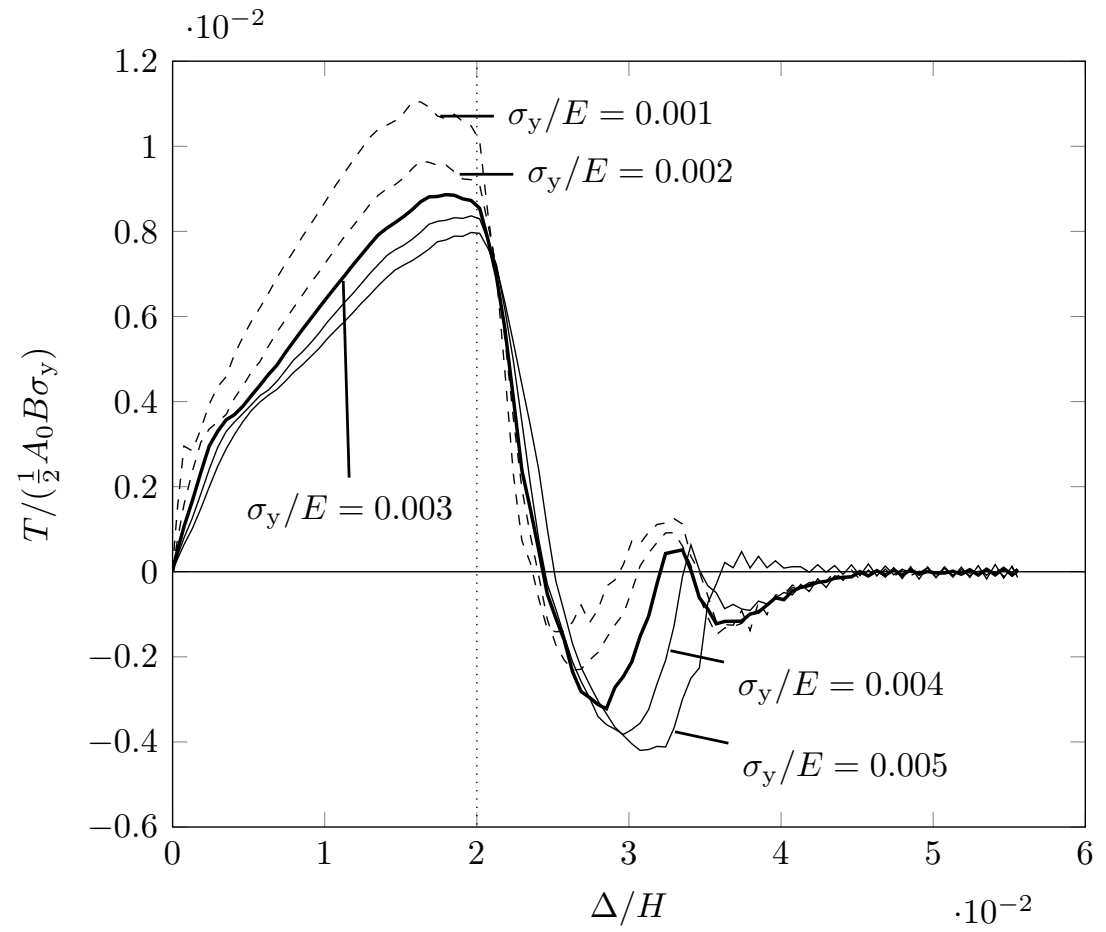

Figure 13: Normalized torque for different initial yield strains $\sigma_{\mathrm{y}} / E$. All other parameters are as given in Table 1. The vertical dotted line indicates the event where the imposed torsional loading changes sign. 

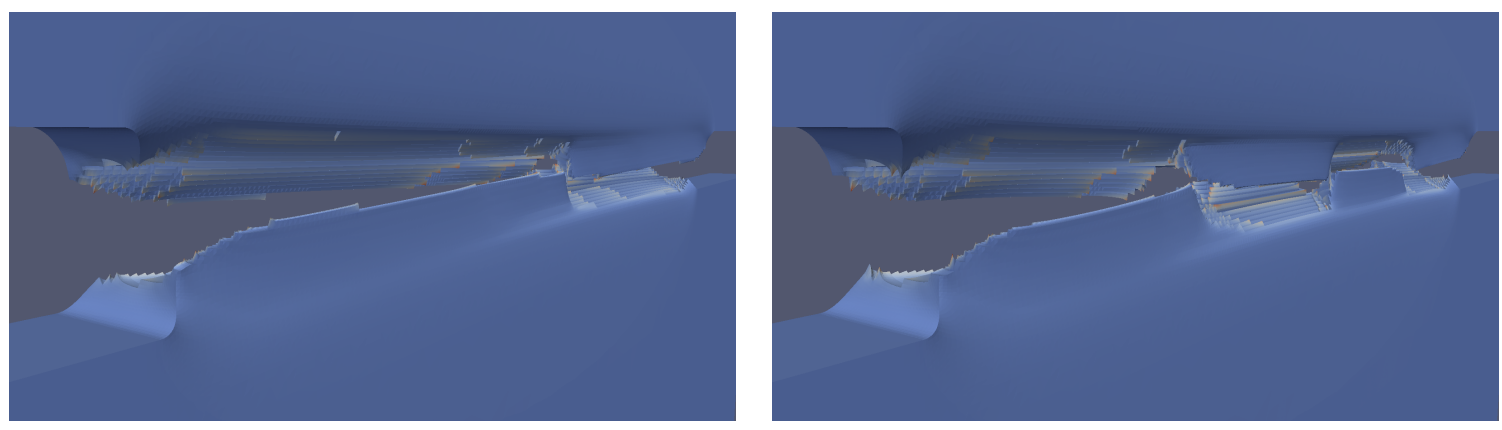

(a) $N=0.04$

(b) $N=0.045$
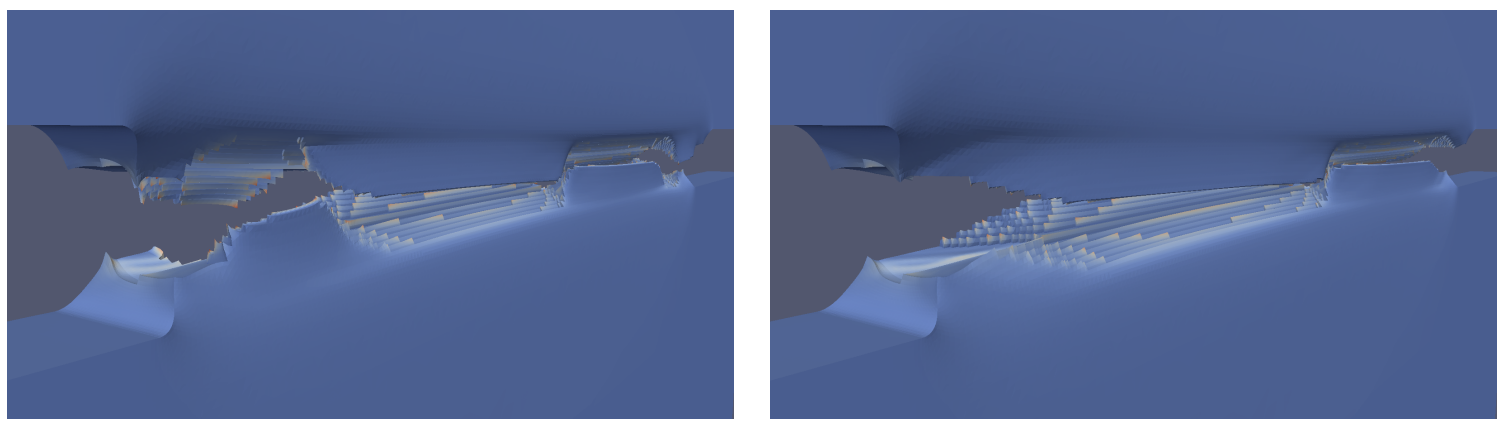

(c) $N=0.055$

(d) $N=0.06$

Figure 14: Crack surface for different strain hardening exponents. Other model parameters are as given in Table 1. 


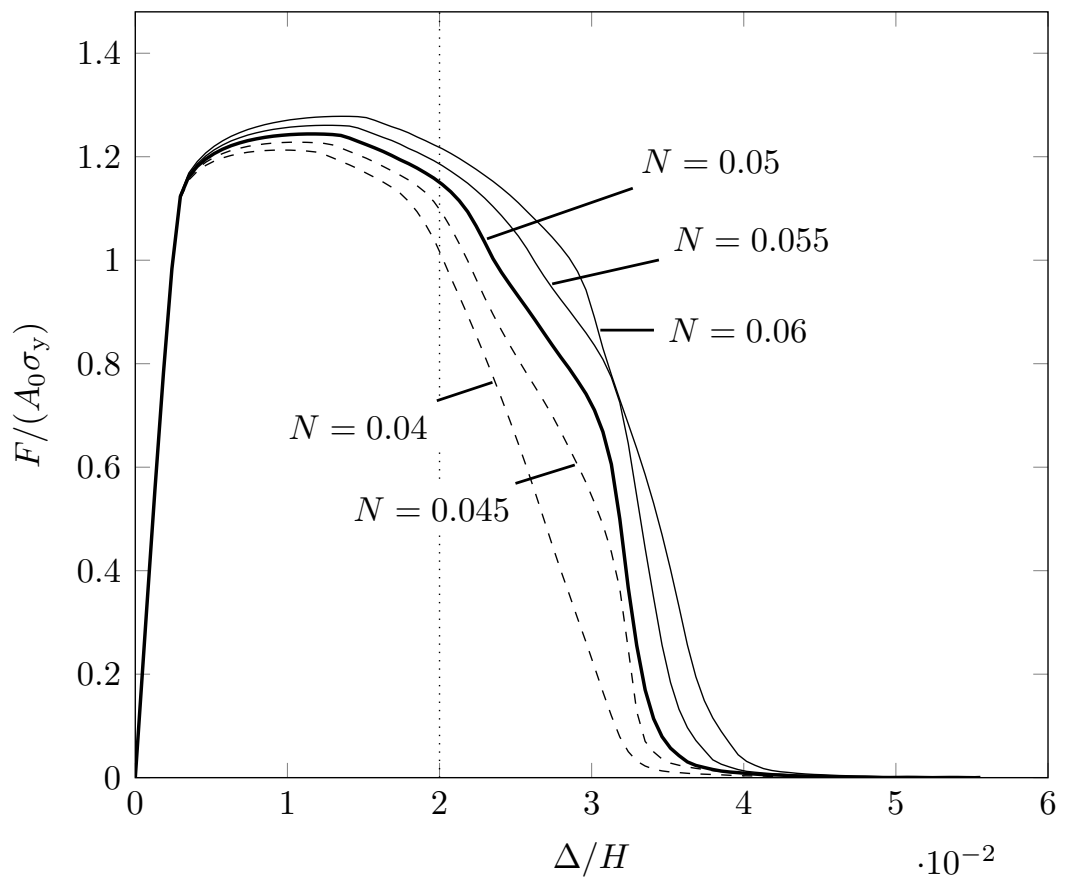

Figure 15: Normalized tensile force for different values of strain hardening exponent $N$. All other parameters are as given in Table 1. The vertical dotted line indicates the event where the imposed torsional loading changes sign.

will promote the crack tip flip. However, if either parameter becomes too small or too large the crack tip flip is absent. In these cases a slant fracture, perhaps with a leading cup-cup fracture morphology, will occur because damage evolves so fast (or so slow) that only the first positive torsional load (or the second negative torsional load) will markedly affect the damage level in the ligament.

Compared to the strain hardening and the void volume fraction, initial yield strain $\sigma_{\mathrm{y}}$ has a somewhat weaker effect, although increasing the value will indeed delay the crack tip flip. Stated differently, the initial yield strain will affect the timing of crack tip flipping and only outside a relatively large interval will it prevent crack tip flipping from happening.

All torque curves presented indicate a change of slope that corresponds to the 


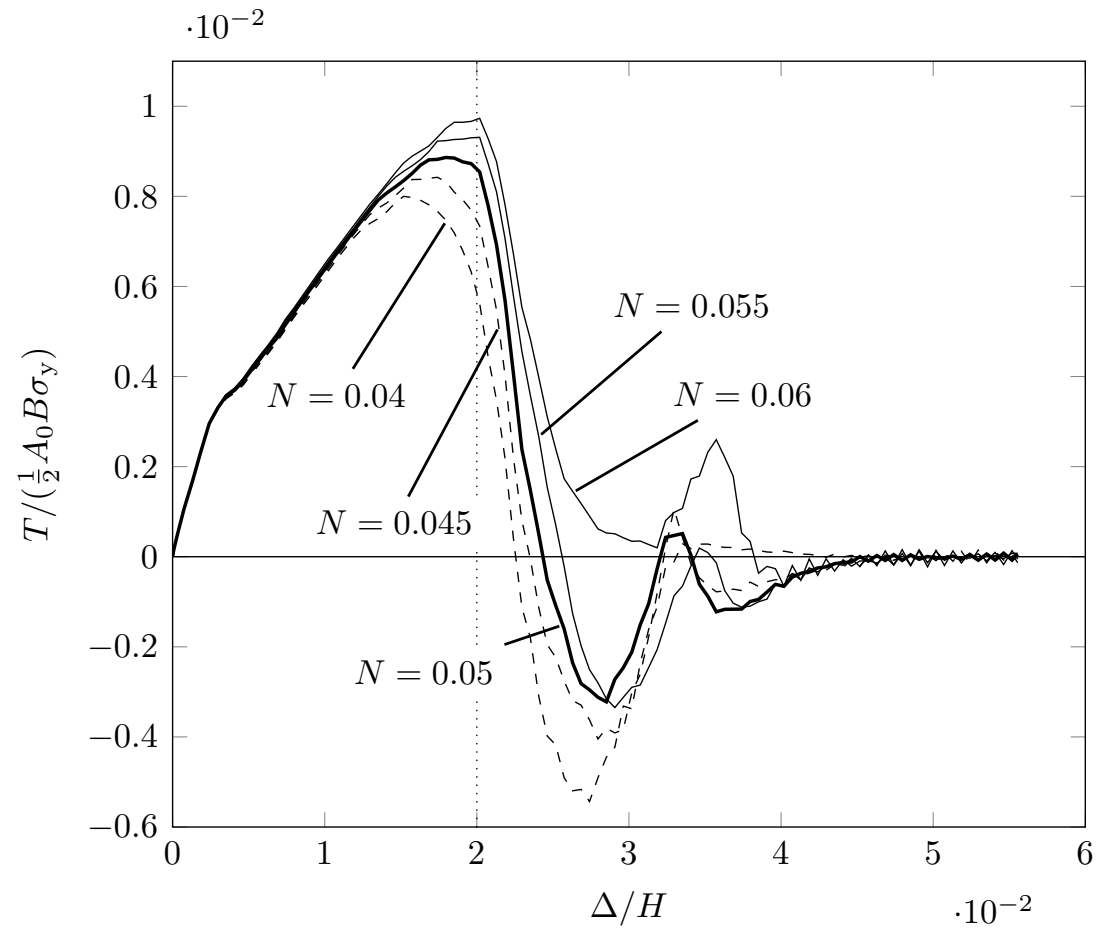

Figure 16: Normalized torque for different values of strain hardening exponent $N$. All other parameters are as given in Table 1. The vertical dotted line indicates the event where the imposed torsional loading changes sign. 
change in sign of the prescribed torsional deformation. It seems remarkable that the crack tip flip is not directly visible in the torque curves, but this result is consistent with the experimental results of Jensen (2015). It has been noticed that many of the torque curves experience a small bump in the response (near $\Delta / H=0.033$ ), however, this part is related to the fracture of the ligament itself and not the crack tip flipping.

The tensile force curves consistently show a (small) characteristic feature when crack tip flipping takes place. For example, the curves corresponding to $f_{0}=0.01$ and $f_{0}=0.012$ in Fig. 9 show a drop near $\Delta / H=0.024$, and these are the only cases with crack tip flipping for varying $f_{0}$. It can be seen that the tensile force recovers when flipping occurs, and carefully going through the experimental work of Jensen (2015) this effect was also observed in the measured curves.

Topics for future work include parametric studies changing parameters what were held constant in this work. Also, an investigation of how the dimensionless groups (possibly) interplay with each other would advance the understanding of crack tip flipping.

\section{Acknowledgments}

The work is financially supported by the VILLUM FOUNDATION Young Investigator Programme in the project "A New Phenomenon Yet to be Resolved in ductile PLATE tearing", grant VKR023451. The authors acknowledge the fruitful discussion with Dr. Pawel Woelke, Thronton Tomasetti Inc. and mechanical engineer Lasse Specht Jensen, Burmeister and Wain Energy A/S, is greatly acknowledged for his work on lab experiments. 


\section{References}

Budiansky, B., 1964. Remarks on Theories of solid and structural mechanics. Harvard University, SIAM, 1517-1541.

El-Naaman, S., Nielsen, K., 2013. Observations on Mode I ductile tearing in sheet metals. Europ. J. Mech. A/Solids 42, 54-62.

Gabriel, E., Fagg, G. E., Bosilca, G., Angskun, T., Dongarra, J. J., Squyres, J. M., Sahay, V., Kambadur, P., Barrett, B., Lumsdaine, A., Castain, R. H., Daniel, D. J., Graham, R. L., Woodall, T. S., September 2004. Open MPI: Goals, concept, and design of a next generation MPI implementation. In: Proceedings, 11th European PVM/MPI Users' Group Meeting. Budapest, Hungary, pp. 97-104.

Gruben, G., Hopperstad, O., Børvik, T., 2013. Simulation of ductile crack propagation in dual-phase steel. Int. J. Frac. 180, 1-22.

Hickey, W. F., Ravi-Chandar, K., August 2015. Transition from flat to slant fracture in ductile materials. Springer International Publishing, Ch. 9, pp. 215-235.

Hutchinson, J., 1973. Finite srain analysis of elastic-plastic solids and structures. Harvard University, AMD, 17-29.

Jensen, L., 2015. Experimental and numerical study of mixed mode i/iii crack propagation. Master's thesis, Technical University of Denmark.

Karypis, G., Kumar, V., 1999. A Fast and Highly Quality Multilevel Scheme for Partitioning Irregular Graphs. SIAM Journal on Scientific Computing 20, 359-392. 
Nielsen, K., 2008. 3D modelling of plug failure in resistance spot welded shear-lab specimens (DP600-steel). Int. J. Fract. 153, 125-139.

Nielsen, K., 2010. Predicting failure response of spot welded joint using recent extensions to the Gurson model. Comp. Mater. Sci. 48, 71-82.

Nielsen, K., Gundlach, C., 2016. Crack Tip Flipping under Mode I Tearing: Investigated by X-Ray Tomography. (submitted for publication).

Pardoen, T., Hachez, F., Marchioni, B., Blyth, P., Atkins, A., 2004. Mode I fracture of sheet metal. J. Mech. Phys. Solids 52, 423-452.

Pineau, A., Benzerga, A., Pardoen, T., 2016. Failure of metals I: Brittle and ductile fracture. Acta Materialia 107, 424-483.

Rivalin, F., Pineau, A., Di Fant, M., Besson, J., 2001. Ductile tearing of pipeline-steel wide plates - I. dynamic and quasi-static experiments. Eng. Frac. Mech 68, 329-345.

Simonsen, B., Törnqvist, R., 2004. Experimental and numerical modelling of ductile crack propagation in large-scale shell structures. Marine Struc. 17, 1-27.

Springmann, K., Kuna, M., 2005. Identification of material parameters of the gursontvergaard-needleman model by combined experimental and numerical techniques. Computational Materials Science 33, 501-509.

Tvergaard, V., 1982. Influence of void nucleation on ductile shear fracture at a free surface. J. Mech. Phys. Solids 30, 399-425.

Tvergaard, V., 1990. Material failure by void growth to coalescence. Adv. Appl. Mech. $27,83-151$. 
Tvergaard, V., Needleman, A., 1984. Analysis of the cup-cone fracture in a round tensile bar. Acta Metall. 38, 157-169.

Xue, Z., Pontin, M., Zok, F., Hutchinson, J., 2010. Calibration procedures for a computational model of ductile fracture. Engineering Fracture Mechanics 77, 492-509.

Zheng, L., Petry, D., Rapp, H., Wierzbicki, T., 2009. Mode I fracture of large-scale welded thinwalled AA6051 extruded panels. Thin-Walled Structures 47, 375-381. 